Cipango Cahiers d'études japonaises

$15 \mid 2008$

Guerre, colonialisme et commémoration

\title{
Pour réintégrer le Japon au sein de l'histoire mondiale
}

Histoire de la colonisation et guerres de mémoire

To Bring Japan Back into World History: History of Colonization and War of

Memories

Arnaud Nanta

\section{(2) OpenEdition}

Journals

Édition électronique

URL : https://journals.openedition.org/cipango/100

DOI : $10.4000 /$ cipango. 100

ISSN : 2260-7706

Éditeur

INALCO

Édition imprimée

Date de publication : 1 janvier 2008

Pagination : 35-64

ISBN : 978-2-85831-177-4

ISSN : $1164-5857$

Référence électronique

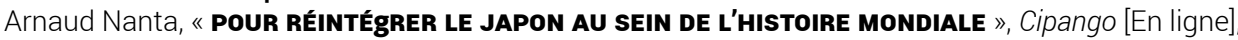

15 | 2008, mis en ligne le 11 novembre 2011, consulté le 30 juin 2021. URL : http://

journals.openedition.org/cipango/100; DOI : https://doi.org/10.4000/cipango.100

Ce document a été généré automatiquement le 30 juin 2021.

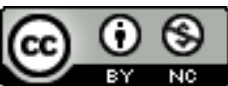

Cipango est mis à disposition selon les termes de la Licence Creative Commons Attribution - Pas d'Utilisation Commerciale 4.0 International. 


\title{
Pour réintégrer le Japon au sein de l'histoire mondiale
}

\author{
Histoire de la colonisation et guerres de mémoire \\ To Bring Japan Back into World History: History of Colonization and War of \\ Memories
}

Arnaud Nanta

Socialement, politiquement et juridiquement parlant, il
y avait des différences très marquées entre les plaines
[du Nouveau Monde] et [...] celles de l'Europe. [...] Pour
l'historien, il est tout aussi important de ne pas
négliger leurs points communs que de tenir compte de
leurs différences. ${ }^{1}$

1 L'histoire de la colonisation japonaise soulève une controverse historique et surtout mémorielle récurrente depuis le début de la décennie 1980. Cependant, souvent traitée pour elle-même, cette situation extrême-orientale oblige en réalité à s'éloigner du Japon pour, plutôt, analyser la dynamique de la colonisation moderne à l'échelle de la planète d'une part, et, d'autre part, la façon dont sa mémoire s'est construite de façon globale au sein de tous les pays concernés. Cela ne pourra être fait qu'en considérant l'ensemble des anciennes puissances coloniales aujourd'hui lieux de débats similaires. Soixante ans se sont écoulés depuis la fin de la colonisation japonaise - au Japon, la fin de la Seconde Guerre mondiale et la décolonisation se sont superposées - ce qui permet de nombreuses comparaisons avec les pays européens (notamment la Grande-Bretagne, les Pays-Bas ou la France qui menèrent des guerres coloniales après $1945^{2}$ ), au lieu de traiter le cas du Japon séparément des colonisations occidentales, en le saisissant dans une "spécificité" empiriquement fragile, comme le font nombre d'historiens occidentaux. Une telle perspective historique transnationale des colonisations modernes ne permettrait-elle pas d'en saisir les dynamiques et les blocages contemporains, et, au-delà, ne contribuerait-elle pas à une meilleure compréhension de l'histoire mondiale? 
2 On peut craindre en effet que ne pas saisir ces histoires (et mémoires) croisées dans le contexte géopolitique international d'émulation des puissances et États nations modernes qui fut le leur, n'empêche de saisir la dynamique commune à tous ces empires coloniaux issus $\mathrm{du} \mathrm{xIX}^{\mathrm{e}}$ siècle. De cela découlent des réductions récurrentes concernant le Japon, telle la focalisation sur la Seconde Guerre mondiale et la violence, parfois avec des arguments culturalistes, ou encore la comparaison systématiquement faite avec l'Allemagne : autant d'interprétations qui, justement, passent outre le passé colonial japonais en tant tel et en tant que phénomène mondial, non spécifique à un pays et irréductible à la Seconde Guerre mondiale ${ }^{3}$. Saisie au $\mathrm{xIx}^{\mathrm{e}}$ et au début $d u \mathrm{xx}^{\mathrm{e}}$ siècle, la colonisation japonaise ne sera-t-elle pas mieux comprise en étant pensée en système avec celle des pays susmentionnés? Et cela ne vaut-il pas également pour les problèmes mémoriels auxquels nous sommes confrontés aujourd'hui ?

3 En face, il est vrai que d'autres a priori répondent, en Asie orientale, à ces a priori occidentaux. D'abord, il y a ceux qui considèrent la modernité japonaise comme " anormale ", car colonisatrice, alors pourtant qu'une telle lecture semble impossible au vu de l'histoire de la colonisation européenne. Ensuite existe la croyance selon laquelle, à la différence de l'Asie orientale, les mémoires seraient apaisées en Europe. Or celles-ci ne le sont pas: si un "travail de mémoire » a bien été réalisé entre les puissances, les sociétés européennes se sont en revanche largement désintéressées de la question coloniale après les décolonisations.

Plutôt que de revenir sur les systèmes coloniaux dans leurs détails concrets, le présent article tentera de montrer en quoi la colonisation moderne - qui peut être considérée comme un type particulier d'impérialisme (c'est-à-dire un type d'expansion et de domination) qui concrètement a soumis des populations saisies comme des altérités irréductibles et présupposait la supériorité militaire absolue de la métropole ${ }^{4}$ - a été une domination systématique exercée par les puissances qui se sont formées au XIX siècle, et comment elle a constitué une seule et unique dynamique mondiale dont le Japon faisait partie intégrante en tant qu'État-nation constitué lui aussi à cette époque. Nous proposons donc de reformuler le débat historique sur la colonisation japonaise afin de remettre celle-ci en perspective au sein de la dynamique coloniale mondiale, en insistant sur trois axes centraux qui montreront pourquoi elle ne peut être considérée isolément.

5 Nous nous pencherons dans un premier temps sur l'historiographie japonaise de la colonisation et sur l'importance d'une réflexion sur la perception de la Chine à l'époque, en montrant comment, dans le cas du Japon, se sont entrechoquées colonisation et compétition avec les puissances impérialistes 5 .

6 Nous reviendrons ensuite sur les débats politiques et historiques récents concernant la colonisation japonaise, afin de montrer leur dimension en réalité générale au-delà des $a$ priori sur le Japon.

7 Enfin, dans un dernier temps, nous tenterons trois remarques de portée générale sur la colonisation autour des cas de la France et du Japon, afin de dépasser les discours sur la spécificité et tenter de mieux saisir la nature de cette dynamique mondiale que fut le fait colonial moderne au travers de quelques caractéristiques concrètes ${ }^{6}$. 


\section{L'Histoire, la mémoire et la question de la perception de la Chine}

De même qu'il faut distinguer entre les deux dynamiques que constituent d'une part la Guerre de l'Asie et du Pacifique (1937-1945) et, d'autre part, la colonisation (japonaise), il est également essentiel de distinguer les travaux historiques portant sur ces deux corps d'événements historiques. Les premiers ont débuté au Japon dès l'après-guerre, tandis les seconds ont débuté vers 1965. À titre de repère, notons que l'historiographie française sur la colonisation (française) se mit en place vers la fin des années 1970 et autour du début des années 1980, c'est-à-dire également environ vingt ans après la fin de la décolonisation $(1962)^{7}-l^{1}$ 'historiographie japonaise sur la colonisation est ainsi, contrairement à une idée reçue, d'un volume incomparablement supérieur à, par exemple, l'historiographie française sur l'histoire de la colonisation française. Nous poserons ici quelques repères sur la chronologie de cette recherche, pour ensuite réfléchir sur l'ambiguïté de la nature de la pénétration en Chine ${ }^{8}$.

9 La Guerre de l'Asie et du Pacifique fut décrite de façon systématique notamment par Inoue Kiyoshi 井上清 (1913-2001), historien anti-impérialiste majeur des décennies 1950-1970, qui mit en avant dès la fin du conflit la relation entre «système impérial » en métropole et impérialisme expansionniste, dans Nihon no gunkokushugi 日 本の軍国主義 (Le militarisme japonais) ou Tennō-sei 天皇制 (Le système impérial) ${ }^{9}$ c'est-à-dire en se focalisant sur le régime politique japonais pensé en imbrication avec l'expansion extérieure. En revanche, à la suite d'une littérature romanesque sur l'époque coloniale ${ }^{10}$ ou d'écrits de rapatriés, l'historiographie japonaise de la colonisation japonaise débuta, vingt ans après la décolonisation, par exemple avec Kobayashi Hideo 小林英夫 (né en 1943) en histoire économique (notamment sur la Corée, Hong Kong et la Mandchourie) ${ }^{11}$, avec Yamabe Kentarō 山辺健太郎 (1905-1977), auteur d'études systématiques sur l'annexion de la Corée ${ }^{12}$ et sur la colonisation - si l'on excepte l'historiographie datant de la période coloniale, l'étude de Yamabe Nihon tōchi-ka no Chōsen 日本統治下の朝鮮 (La Corée sous le gouvernement du Japon) ${ }^{13}$ est, à notre connaissance, la première étude systématique sur ce sujet -, avec le spécialiste d'histoire militaire Ōe Shinobu (né en 1928) qui contribua à recentrer l'histoire de la fin du xix ${ }^{e}$ siècle depuis le Japon et l'Europe ${ }^{14}$ vers l'Asie avec Kindai Nihon to Ajia 近代日本 とアジア (Le Japon moderne et l'Asie) en 1968 ${ }^{15}$, et qui dirigera en 1992-1993 une grande synthèse sur la colonisation japonaise, couronnant un quart de siècle de recherche ${ }^{16}$, ou encore avec la constitution d'un corpus d'archives relatives à l'annexion de la Corée dès les années $1970^{17}$, très enrichi deux décennies plus tard.

Cette historiographie sur la colonisation est déjà présente dans la seconde édition de 1962-1964 des Cours Iwanami d'histoire du Japon et occupe une place conséquente dans la troisième édition de 1975-1977 ${ }^{18}$. Au même moment, la Chōsen shi kenkyūkai 朝鮮史研 究会 (Société [japonaise] d'histoire de la Corée), fondée en 1959, qui étudie, comme son nom l'indique, l'ensemble de l'histoire de la péninsule coréenne, consacrait dès les débuts de son activité une place conséquente à la colonisation dans son bulletin annuel. Tout comme en France pour la colonisation de l'Algérie ${ }^{19}$, une partie importante de ces premiers chercheurs s'étaient mis à étudier l'histoire de la Corée parce qu'ils y étaient nés, ce qui leur valut d'être considérés comme ayant «trahi » le groupe plus général des rapatriés. À ce sujet, le cas de l'historien Hatada Takashi 旗田巍 (1908-1994), qui occupe une place importante au sein de l'historiographie critique des années 
1960-197020, ou celui de Yamabe, déjà mentionné, sont emblématiques au même titre que celui de Claude Liauzu en France.

11 Le travail historique japonais sur la guerre - qui n'est pas le sujet du présent article posa un double problème dès la fin des années $1950^{21}$, tout comme le fera l'historiographie de la colonisation au début des années 1980. Ce qui importe pour nous ici est, le fait que, majoritairement, les historiens et intellectuels japonais de la première phase (histoire de la guerre), notamment les libéraux et les marxistes, ont interprété les institutions d'avant-1945 comme " militaristes », cette période étant mise en vis-à-vis avec un système d'après la guerre affirmé comme "vraiment démocratique $»^{22}$ : cette interprétation de l'avant-guerre, historiquement fausse ${ }^{23}$, contribua à fixer le regard historique sur la période 1937-1945 voire 1931-1945 comme apothéose d'un «totalitarisme » imaginé de l'avant-guerre, tout en éclipsant l'empire colonial de par une sur-focalisation sur la nature du régime, c'est-à-dire sur le «système impérial». Cette reconstitution idéologique du passé du Japon a perduré dans ce pays et se trouve, de plus, largement partagée par les chercheurs en sciences humaines des pays occidentaux. Autrement dit, la superposition entre impérialisme colonial et "système impérial» a été autant le fait d'historiens occidentaux que japonais après 1945, ceux-ci ayant saisi le Japon de 1868 à 1945 comme un bloc cohérent tout en mettant en exergue une sorte de « projet » initial conquérant de l'État de Meiji. On peut qualifier cette lecture de l'histoire de monolithique et d'intentionnaliste, quand elle ne réactive pas chez les Occidentaux une sorte d'orientalisme au sens d'Edward Saïd, c'est-à-dire une essentialisation des particularités ${ }^{24}$.

12 Ainsi, ces critiques d'avant 1970 contre le "militarisme» concernaient les responsabilités de l'État japonais vis-à-vis de la population japonaise ${ }^{25}$ sans aborder la question coloniale ${ }^{26}$. La mémoire, comme la recherche, étaient globalement dominées par une critique du « système impérial » et des effets purement nationaux du régime du temps de guerre (semblablement, la question coloniale disparaissait - à considérer qu'elle eût été importante - chez d'autres pays où l'on ne pensait pas de façon imbriquée le régime national et l'expansion extérieure, tel le cas de la France républicaine et coloniale ${ }^{27}$ ). La colonisation n'était en fait à l'ordre du jour chez aucune puissance de l'époque. Au Japon, cette "absence » s'explique aussi par des pressions franco-britanniques lors du Procès de Tōkyō, parce que la colonisation n'est pas faite de guerre $^{28}$, et plus largement par son " oubli nécessaire » dans la mémoire collective, qui dérive de la mémoire des bombardements - véritable "mémoire-écran » - et du trauma lié à la brusque perte de l'empire colonial. Semblablement l'Algérie disparut durant presque quarante ans des débats publics en France, malgré la permanence de la controverse sur la torture, et l'on devrait se demander quel aurait été le choc si la France avait été brusquement, et irrémédiablement, ramenée à son imperium purement national d'un seul coup en $1945^{29}$.

13 Pour ces raisons, nous pensons que le débat mémoriel actuel, en Asie orientale comme en Europe occidentale, constitue surtout un legs de l'époque coloniale, autrement dit qu'il traduit des tensions ou un déphasage entre anciennes puissances coloniales et expays colonisés. En Europe comme au Japon, ce dont la reconnaissance fut exigée, et ce qui fut jugé durant la seconde moitié du $\mathrm{xx}^{\mathrm{e}}$ siècle, c'est la guerre entre puissances, tandis que jusque dans des années récentes (mais, donc, plus tôt pour le Japon) la colonisation ne fut, elle, jamais remise en cause, jamais jugée par les anciennes puissances, malgré des critiques formulés vis-à-vis de la SDN, puis au sein de l'ONU - on reviendra sur le cas de 
la France plus $\operatorname{bas}^{30}$. Or il est essentiel d'avoir à l'esprit que ni la Corée, ni la Chine n'étaient des puissances au début du $\mathrm{xx}^{\mathrm{e}}$ siècle. S'opposent ainsi l'exemple francoallemand d'une part et, d'autre part, les tensions entre l'Allemagne et la Pologne - cet exemple pris à l'ouest du continent est éclairant: en 1939/40-1945, la France fut occupée, mais la Pologne fut colonisée -, ou celles entre la France et l'Algérie, ou encore celles entre le Japon et la Corée, voire la Chine. Si nous relions ainsi questions mémorielles et colonisation, c'est aussi en constatant l'absence de conflit mémoriel, par exemple, entre le Japon et la Russie ou les États-Unis, pays qui se sont combattus au $\mathrm{xx}^{\mathrm{e}}$ siècle. Mais ce qui pose problème ici, dans notre perspective, cela n'est pas la Corée ou Taïwan (c'est-à-dire l'ancien empire colonial, constitué en 1895-1905), mais plutôt la Chine.

La place de la République de Chine (Zhōnghuá Mínguó 中華民國; la future Chine du Guómíndăng 國民黨, fondée en 1912) est, de fait, problématique. Celle-ci fut assurément un pays belligérant ${ }^{31}$ après la fin de la période dite des seigneurs de la guerre - qui démarre en 1916 pour aller jusqu'aux années 1930 -, et la guerre, ou plutôt les guerres menées par le Japon sur le continent à partir de 1928-1931 sont elles aussi d'évidence des « conflits militaires ». Mais relèvent-elles réellement de la guerre entre puissances ou bien de la colonisation, ou plus précisément de la poursuite d'un effort colonial ? Pour comprendre les perceptions actuelles du passé, il est nécessaire de saisir avec précision la perception japonaise et mondiale de la Chine entre 1911 et 1928-1931, et donc de préciser la perspective historique selon laquelle la Chine doit être abordée : car le concept d'«années trente» ne peut pas expliquer une «brusque " agression japonaise contre la Chine, agression en réalité indissociable d'une avancée continue, remontant à 1895-1905. Autrement dit, il faudrait davantage considérer l'« Incident de Mandchourie » de 1931 comme l'aboutissement d'un processus de consolidation de l'empire colonial sur le continent, plutôt que comme un point de départ vers ce qui suivra, selon une lecture téléologique de l'histoire, et cela sans toutefois voir d'« essence impérialiste » dans l'État japonais issu de Meiji.

Au-delà d'une continuité des tensions géopolitiques en Asie orientale, comme ailleurs, durant la première moitié $\mathrm{du} \mathrm{xx}^{\mathrm{e}}$ siècle, il convient, là encore, de distinguer le mouvement colonialiste japonais de la dynamique propre à la Seconde Guerre mondiale. ${ }^{32}$ Non seulement on ne peut appliquer la chronologie ouest-européenne, notamment celle suivant la Crise de 1929, au reste du monde, mais, surtout, il convient de ne pas distordre des temporalités qui sont pour nous déjà éloignées en " compressant » le temps et en passant à côté de la courte durée qui sépare la fin de la Première Guerre mondiale (et le monde de cette époque) de "l'incident de Mandchourie » - treize ans est un intervalle court, juste deux présidences sous la v République.

Pour ce qui importe ici, la pénétration japonaise en Chine et en Corée relève du mouvement impérialiste mondial $\mathrm{du} \mathrm{xIX}^{\mathrm{e}}$ siècle, époque où toutes les puissances européennes (Russie tsariste comprise) avaient placé la Chine dans une relation semicoloniale, après les deux guerres de l'opium (1839-1842 et 1856-1860) ou après les deux guerres franco-chinoises (1883, puis 1884-1885) pour le contrôle du Viêtnam et la fondation de l'Union indochinoise (1887). Comme l'a noté le spécialiste de la colonisation Christopher A. Bayly, dans une étude très critique à l'encontre de l'historiographie occidentalo-centrée: "Il est clair que lorsque les corps expéditionnaires européens et américains envahirent la Chine sous prétexte de mater 
la rébellion des Boxers en 1900, seules leurs rivalités empêchèrent que la Chine ne soit dépecée comme l'avait été l'Afrique. ${ }^{33}$ "

La perspective exposée ci-dessus vis-à-vis de la Chine du début $\mathrm{du} \mathrm{xx}^{\mathrm{e}}$ siècle non seulement permet d'expliquer les tensions mémorielles actuelles, mais permet aussi de saisir plus exactement les perceptions qui avaient cours à l'époque coloniale. Les puissances (Japon compris) intervenaient militairement en Chine encore au début du $\mathrm{xx}^{\mathrm{e}}$ siècle, et $\mathrm{y}$ conservaient des comptoirs et même des colonies pour la Russie et pour l'Allemagne, pays parti en retard dans la compétition colonialiste (cf. infra partie 3). Le Japon était alors l'allié de la France et de la Grande-Bretagne et son expansion de 1914 fut réalisée aux dépens de la colonisation allemande, avalisée par la Société des Nations après la guerre ${ }^{34}$; il ne déroba pas la Mandchourie à la Chine en 1931, mais à la Russie, qui l'occupait au début du siècle, par à-coups après sa victoire de 1905 à partir de la région du Kwantung 關東洲 (Guāndōngzhōu), en obtenant des concessions autour des lignes de chemin de fer, tout comme le faisaient les Européens ailleurs ${ }^{35}$. A-t-on bien remarqué que la destitution forcée de l'empereur Than Thai 成泰 au Viêtnam par les Français avait eu lieu exactement la même année que celle de l'empereur ${ }^{36} \mathrm{Kojong}$ 高宗 (고종) en Corée par les Japonais, en 1907 ?

Le grand mouvement de colonisation en Chine et en Corée, et au-delà en Asie du NordEst et du Sud-Est, concernant l'ensemble des puissances ${ }^{37}$, selon quels critères pouvonsnous analyser ce qui se produisit en Chine "après » 1931 ? À cette époque, l'Empire colonial japonais stricto sensu (l'île de Taïwan et la Corée) était consolidé depuis déjà un quart de siècle. Comment expliquer qu'un même mouvement de domination en Asie orientale - la dynamique de la colonisation - soit tantôt considéré comme relevant de la «colonisation» lorsqu'il est le fait des Européens, tantôt comme relevant du «militarisme» lorsqu'il est le fait du Japon $?^{38}$ Comme nous l'avons noté en introduction, la colonisation est avant tout un type de domination, et non pas la domination d'une « race » sur une autre - pure opinion qui interdirait historiquement au Japon d'être colonisateur en Asie, ou à l'Allemagne en Pologne..$^{39} \mathrm{Ce}$ qui importe ici est le fait que, bien que soi-disant objet d'une politique d'assimilation - qui aurait pu être réalisée à terme, mais là n'est pas le débat -, les populations dominées (en Corée, en Algérie ou en Pologne) étaient en réalité l'objet d'un regard réifiant les saisissant comme une altérité irréductible à la nation de métropole, ce qui était contradictoire avec la velléité assimilatrice. Les discours légitimant la colonisation sur le thème de la mission civilisatrice ont été très largement communs à toutes les puissances, parce qu'ils cherchaient à en démontrer la supériorité inhérente : cela devrait plutôt amener à analyser de façon globale cet "universalisme » des dominants, en opposition à un universalisme réel jamais réalisé ${ }^{40}$. Le traitement des Coréens, et des Coréennes, durant la Seconde Guerre mondiale montra ce qu'il en était concrètement de la réalité de l'«assimilation», tout comme l'a fait le refus continu de conférer la citoyenneté en Algérie jusqu'en 1962.

\section{La colonisation et sa mémoire : des questions mondiales}

19 Ce problème de la conception de l'autre ne peut être éludé, car si celle-ci n'est pas en soi le moteur de la colonisation moderne (dont les causes sont plutôt à rechercher dans la compétition interétatique), elle permet d'en comprendre le fonctionnement, ainsi 
que les blocages. La perception, passée et présente, des événements passés (qui sont parfaitement isolables) possède un rôle central dans les actuelles "guerres de mémoire », ici dans les responsabilités soulevées par les deux Corée et par la Chine. La dénomination des événements et des situations n'est pas neutre. Qu'il s'agisse de "guerre» entre des "grands États» (entre "puissances", selon le mot forgé au Congrès de Vienne), et dans ce cas les responsabilités devront être admises et donner lieu, suivant le "modèle allemand" pour le national-socialisme, à une repentance publique et à un "travail de mémoire ». Mais qu'il s'agisse de "colonisation », et dans ce dernier cas, soit les anciennes métropoles « oublient » comme si rien n'avait eu lieu (ni «femmes de réconfort ", ni massacre de Nánjīng [Nankin] en 1937-1938 pour le Japon, ni les massacres de Sétif en 1945 et de Madagascar en 1947, ni la torture en Algérie lors de la « bataille d'Alger » en 1957 pour la France), soit un discours officiel sur les « aspects positifs de la colonisation » peut même avoir libre cours ${ }^{41}$. S'opposent ainsi la «vraie guerre» et les "événements»: la Guerre d'Algérie (1954-1962) n'a été reconnue comme "guerre » qu'en 1999 par la République française, mais jusque-là, il ne s'agissait que des "événements d'Algérie ", de même que l'on évoquait au Japon les " événements de Corée » (jiken 事件 ou jihen 事変) ou les " événements de Chine ", justement.

Pour cette raison, le débat récurrent depuis 1910 sur la nature de la présence japonaise en Corée - colonisation ou « occupation »? - ne doit pas être sous-estimé : il y a ici une lutte concernant la dénomination, qui ne peut être bien comprise que dans le cadre des enjeux décrits ci-dessus ${ }^{42}$. Parallèlement, Paris et Tōkyō continuent à commémorer parmi les «morts pour la France» ou «morts pour le Japon $»^{43}$ les militaires tombés lors des guerres coloniales : le sanctuaire Yasukuni ou l'Arc de triomphe sont des lieux de mémoire consacrés à tous les morts pour la patrie - l'empire colonial se superposant ici à la "patrie». Ces deux lieux ont été fondés dans des contextes analogues, par Napoléon pour les guerres révolutionnaires et napoléoniennes pour le premier, en 1869 suite à la première guerre civile pour le second ${ }^{44}$.

21 Revenons aux débats mémoriels entre le Japon et ses voisins, souvent présentés comme «spécifiques » au premier ou bien comme dérivant de la persistance d'un ressentiment " asiatique » en Chine et en Corée, ce qui dans les deux cas traduit à nouveau la prégnance d'une sorte d'orientalisme au sens de Saïd. Une telle lecture, courante jusqu'à l'explosion du débat colonial en Europe, possède une certaine légitimité si l'on s'interroge sur des groupes aussi particuliers que l'aile dure des conservateurs ou sur l'extrême-droite. Cependant, comme mémoire du fait colonial (que nous discutons ici à propos de la Chine, et qui est indéniable à propos de la Corée), et surtout comme attitude présente d'une ancienne puissance coloniale en continuité avec la période qui a suivi la (dé)colonisation, ce phénomène est généralisable (en 1945-1960, cette attitude condescendante du Japon était approuvée par les autres puissances, encore coloniales) ${ }^{45}$. Précisément parce que l'on ne peut pas superposer guerre et colonisation comme le faisait Aimé Césaire ${ }^{46}$, on doit se demander si, historiquement, le cas du Japon peut réellement être traité à part. Le Japon n'adopte-t-il pas la même position que la France ? Lorsque l'on traite de l'Asie orientale, ne surestime-t-on pas la capacité de la France à se réconcilier avec ses anciennes colonies et notamment l'Algérie?

Cela nous amène au problème suivant : pourquoi la France, et plus largement l'Europe de l'Ouest, sont-elles rattrapées aujourd'hui par ce débat sur le passé colonial, longtemps jugé (depuis 1982) "spécifique» du Japon $^{47}$ ? Comme on l'a noté, la 
décolonisation japonaise s'est achevée plus tôt (en 1945) que celle de la France (achevée en 1962), et en réalité les débats mémoriels ont éclaté plus tôt au Japon (dès 1982) qu'en France (après 2000 environ), suivant ainsi un décalage significatif d'environ vingt années. Une différence pourrait cependant être soulignée dans le débat sur la « responsabilité coloniale » française tel qu'il a lieu depuis l'an 2000 : à la différence du débat homologue au Japon, qui est plus ancien et au sein duquel le clivage gauche/ droite se superpose grosso modo avec le clivage observé au sein du débat sur le passé colonial $^{48}$, dans le cas de la France, en revanche, le débat sur la colonisation traverse le clivage Gauche/Droite. Il a été possible de constater début décembre 2007, lors de la visite du président Nicolas Sarkozy en Algérie, que le Parti socialiste était sur la même ligne que l'ump dans sa façon de saisir l'État français et son honneur "insulté » - on pense aux déclarations du Secrétaire national aux questions internationales, Pierre Moscovici, à propos de "l'autoflagellation » française, à celles du président de la députation socialiste à l'Assemblée nationale et à celles du porte-parole du Parti socialiste ${ }^{49}$. Aucun État n'accepte en réalité de présenter des excuses pour la colonisation, ni n'est prêt à mettre ses criminels coloniaux en jugement, ni à payer des compensations pour fait colonial ${ }^{50}$. Les excuses du président Jacques Chirac le 21 juillet 2005 pour les massacres de Madagascar ont été critiquées par une partie de l'opinion française (lors de son discours du 16 juillet 1995, J. Chirac avait évoqué Vichy, mais pas la colonisation; il précédait de peu les excuses du Premier ministre japonais Murayama Tomiichi 村山富市 du 15 août 1995, qui déclenchèrent une pétition massive du Parti libéral démocrate, alors dans l'opposition ${ }^{51}$ ). Ceci illustre bien le caractère structurel du débat et sa prégnance dans les consciences collectives en Europe et au Japon (on pourrait évoquer la Belgique ou les Pays-Bas) : cette question des mémoires est tout aussi globale que l'est le passé colonial lui-même, irréductible à un pays en particulier.

La recherche menée par les historiens, depuis le milieu des années 1960 au Japon et autour du début des années 1980 en France, a montré quels étaient les traits et caractéristiques des diverses colonisations. Bien évidemment, tout n'a pas été identique en tout lieu, mais, au-delà de particularités historiques évidentes, il semble hasardeux de vouloir mettre en évidence des « spécificités » nationales, voire une opposition entre le système colonial «occidental » et celui du Japon, car ces systèmes relevaient de dynamiques et de logiques homologues. Autrement formulé : s'il est légitime d'étudier les particularités, en revanche la notion de "spécificité " semble hasardeuse en histoire. L'ouvrage précis Histoire des décolonisations au XXe siècle (2006) ${ }^{52}$ discutait aussi, succinctement, des différents systèmes coloniaux et de la période coloniale. On peut cependant s'interroger sur la pertinence d'une opposition tranchée entre les systèmes européens et le cas japonais qui y était faite, le Japon, rangé parmi « les impérialismes extra européens » (dont il est en réalité le seul cas de figure), n'y étant ainsi quasiment pas présenté, tout en voyant pourtant sa colonisation jugée « impitoyable $»^{53}$. Quel est le critère permettant une telle qualification? Et quel est le présupposé de cette distinction?

L'historien Oguma Eiji s'est récemment interrogé sur à la façon la plus pertinente de comparer les différents systèmes coloniaux et sur les limites de telles comparaisons ${ }^{54}$. Nous pensons quant à nous que la seule opposition ou distinction qui fasse sens à l'ère des empires était celle qui existait entre les zones développées du globe et zones qui n'avaient pas accès au "progrès ", ou, autrement formulé, l'opposition entre les puissances et les dominés. En outre, au-delà de différences et de particularités 
concrètes que l'on ne peut bien sûr négliger, nous avons tendance à considérer que tous les systèmes coloniaux modernes étaient assez similaires pour une autre raison, importante, qui est que lesdits empires coloniaux n'ont jamais réellement été "planifiés» ni même pensés, mais ont plutôt été construits par des bricolages successifs, c'est-à-dire dans une improvisation permanente.

Concrètement, bien qu'il soit admis que les Anglais et les Français n'ont pas eu la même perception de "l'évolution des peuples colonisés " ${ }^{55}$, il nous semble impossible de distinguer avec netteté entre un système "anglais indirect » et un système "français direct » : les empires coloniaux ont été pluriformes et ont toujours adopté des solutions légales diverses et variées, et toujours ad hoc (après tout, les constitutions de métropole n'étaient appliquées nulle part $\left.{ }^{56}\right)$; le système d'administration directe est en réalité davantage un résultat qu'un choix initial, système que l'on peut considérer comme un effet résultant de l'âpreté de la résistance à la colonisation. On souligne souvent l'inflexion dans la politique coloniale française après 1918, qui serait devenue plus "pragmatique » après cette date, mais de grandes réalisations avaient-elles été réalisées dans l'Empire français avant la Première Guerre mondiale? La politique d'assimilation «à la française » a-t-elle vraiment été bien mise en œuvre au travers, par exemple, de l'Université indochinoise de Hanoï, ouverte en 1906 pour être fermée deux ans plus tard (réouverte dix ans plus tard, elle resta très élitiste), ou par une $\mathrm{III}^{\mathrm{e}}$ République jugée par les historiens moins soucieuse des intérêts des Algériens que ne l'était Napoléon III peu avant sa chute $?^{57}$

Cette remarque vaut aussi pour la violence coloniale, car à ce sujet on peut considérer que la situation était proche dans tous les pays concernés ${ }^{58}$. La répression du soulèvement de Wùshè (jap. Musha jiken 霧社事件) à Taïwan en 1930 - la plus importante dans l'histoire de la colonisation japonaise avec celle du $1^{\mathrm{er}}$ mars 1919 en Corée - a eu lieu au même moment que la répression de l'insurrection de Yen Bay en Indochine, et dans les deux cas l'armée réprima avec la même sévérité les insurgés, pour lesquels «la terreur s'abat[tit] sur le Nord $^{59}$ » de l'Indochine et qui virent un ratissage systématique des hauts plateaux à Taïwan. Cette synchronie renforce en outre l'idée d'un ébranlement des empires à l'entrée de la décennie 1930, dans un contexte de montée des revendications d'indépendance suite à la Déclaration de Wilson et la création de la SDN.

Pourtant, sur la question de la violence, le même paradoxe à propos du Japon est observable au sein des discours : alors que les chercheurs occidentaux sont aujourd'hui, majoritairement, opposés à la colonisation, on remarque un discours ou une critique vis-à-vis d'une "spécificité » de la violence coloniale japonaise en opposition à une colonisation "occidentale ", qui évoque à nouveau la prégnance d'une certaine vision orientaliste du Japon. Pierre F. Souyri mettait pourtant en garde contre ce danger, en écrivant en 2003 : «Le régime colonial japonais a la réputation en Occident d'avoir été particulièrement brutal. [J'ai montré qu'en effet] cette domination s'est souvent accompagnée d'une grande violence. Mais gare aux préjugés : parce qu'elle n'était pas le fait d'hommes blancs, cette violence ne nous paraît-elle pas plus illégitime encore? Était-elle pourtant d'une essence si différente que celle pratiquée dans les colonies occidentales $?^{60}$ » Aujourd'hui, un demi-siècle après la fin des décolonisations, nous avons besoin de réfléchir sur les empires coloniaux saisis enfin au sein de la dynamique globale qui était celle de l'époque moderne, et non comme des problèmes historiques purement régionaux. 


\section{La colonisation moderne : la durée, la distance et l'État-nation} Pour la question du temps et de la durée, on oppose souvent une colonisation occidentale « longue" à une colonisation japonaise «courte ${ }^{62}$. Mais quelle est cette frontière que l'on dresse entre la courte durée et la longue durée coloniale ? En 1789, la France ne possédait que $40000 \mathrm{~km}^{2}$ de territoires coloniaux, mais en 1912 ce domaine était passé à 11 millions de $\mathrm{km}^{2}$ : ces territoires furent acquis au XIX ${ }^{\mathrm{e}}$ siècle, en réalité surtout lors de la grande poussée impérialiste des années $1880^{63}$. Cela signifie qu'en France aussi, comme au Japon, la construction de l'empire colonial moderne s'est superposée avec la construction de l'État-nation, durant la $\mathrm{III}^{\mathrm{e}}$ République $^{64}$. Pour ce qui est de l'Afrique du Nord : Alger a été occupée dès 1830, mais l'ensemble de l'Algérie ne fut conquise que vers la décennie 1870, soit autour de la date de fondation de la III République $(1870)^{65}$, la Tunisie devint un protectorat en 1881 et le Maroc ne fut rattaché à la France qu'en 1912. Quant aux autres parties de l'empire colonial français, dans les décennies 1870-1880 l'immense espace africain restait à conquérir, tout comme l'Indochine, joyau de l'empire colonial français (les guerres de pacification au Viêtnam durèrent jusqu'en 189766). Non seulement « l'installation française en Indochine ne s'est pas faite d'un seul coup », mais plus encore elle « ne sera pas durable ${ }^{67} »$.

Qu'en est-il des autres pays européens? Les Indes néerlandaises (actuelle Indonésie) n'étaient pas une colonie si ancienne qu'on le croit généralement : leur conquête ne fut totalement achevée qu'à la veille de la Première Guerre mondiale ${ }^{68}$; et que penser de la durée de la colonisation allemande, alors que l'Allemagne perdit toutes ses colonies au début de la Première Guerre mondiale, ce qui fut la plus courte des colonisations modernes (si l'on excepte la colonisation italienne, au milieu des années 1930) ? Quant au Japon, inversement, il était présent militairement à Taïwan dès 1874, et pesait militairement et surtout économiquement sur la Corée dès 1875; car partout la présence économique a précédé la colonisation. Si décalage il y a, celui-ci est minime et en tous les cas non pertinent. À la différence de la colonisation d'Ancien Régime (qui, elle, a bien ses spécificités comme type de colonisation), on peut considérer que toutes les colonisations modernes ont été courtes. Comme l'a écrit Éric Hobsbawm à propos de la dernière phase de la colonisation moderne, qui concerna l'Afrique et l'Asie orientale : «Aussi curieux que cela paraisse, toute l'histoire du colonialisme sur la quasi-totalité du continent africain, depuis les débuts de l'occupation jusqu'à la formation d'États indépendants, tient dans une seule vie - celle de Winston Churchill par exemple (1874-1965). ${ }^{69}$ » 
31 Le deuxième point sur lequel nous souhaiterions insister est celui de la distance géographique entre les métropoles et les colonies. Il s'agit là d'un élément important au XIX ${ }^{\mathrm{e}}$ siècle (même pour des colonies "proches » comme l'Algérie ou la Corée, Taïwan étant à plus de $1000 \mathrm{~km}$ de l'île japonaise de Kyūshū), mais parfois sous-estimé par certains auteurs, non sans un certain anachronisme historique : en effet, que la France ou le Japon fussent des pays mécanisés à la fin du $\mathrm{XIX}^{\mathrm{e}}$ siècle ne signifie pas qu'ils étaient absolument libres de toute entrave technologique ou logistique. En fait, ils l'étaient bien moins que l'Italie en Lybie ou en Éthiopie un demi-siècle plus tard. La distance géographique explique pourquoi les prérogatives exécutives et législatives des résidents généraux étaient si grandes, ou pourquoi dans le cas du Japon les résidents généraux dépendaient directement de l'empereur. Car en cas d'imprévus ou d'“ événements", le Gouvernement général (Sōtokufu 総督府 pour le Japon) devait être à même de réagir de façon prompte et souveraine : ils étaient ainsi " des hommes au pouvoir considérable $e^{70}$, qui régnaient dans le cadre d'un régime de décrets, modifiables et aménageables par dérogation, en dehors du cadre de la constitution ${ }^{71}$.

Cette question de la distance amène cependant une autre question, à laquelle on ne peut répondre ici, mais qui permet d'enrichir (ou de complexifier) ce tableau. Hannah Arendt utilisait dans L'Impérialisme ${ }^{72}$ le concept d'«impérialisme continental » (en opposition avec «l'impérialisme d'outre-mer») pour désigner l'annexionnisme moderne pratiqué au nom d'une proximité ethnico-raciale, notamment le panslavisme russe ou le pangermanisme allemand. Le cas de la Corée, voire de la Mandchourie, compte tenu du discours sur les « origines communes nippo-coréennes » (nissen dōsoron 日鮮同祖論) mis en avant lors de l'annexion de 1910, ou du discours sur la « culture coréo-mandchoue» (mansen bunka 満鮮文化) qui suivit, semble pouvoir être aussi analysé à la lumière de cette grille. Cela montre la complexité d'un cas où impérialisme et colonisation s'entrechoquent du fait de la compétition entre la Russie et le Japon pour le contrôle du Nord-Est asiatique, ce qui amena à recourir aux arguments de l'annexionnisme. Il faut cependant rappeler l'importance des discours du déterminisme géographique en France dans les années 1930, qui invoquèrent la proximité entre la Provence et l'Algérie, ce qui, avec certaines limites, se rapproche là aussi des discours de l'annexionnisme.

Toujours concernant la question de la distance, on oublie parfois à quel point nos propres régimes et sociétés (française, japonaise ou autre), quand on les étudie à la fin $\mathrm{du} \mathrm{XIX}^{\mathrm{e}}$ siècle ou au début $\mathrm{du} \mathrm{Xx}^{\mathrm{e}}$ siècle, constituent une altérité pour notre regard tellement est importante la différence avec nos sociétés actuelles. De même qu'on ne saisit pas toujours bien les difficultés auxquelles ces sociétés faisaient face durant leur processus de modernisation. Madeleine Rebérioux écrivait à ce sujet: «Il y aurait grande injustice à rendre la République radicale [au début $\mathrm{du} \mathrm{xx}^{\mathrm{e}}$ siècle] responsable de ces mœurs qui, dans les affaires coloniales où la distance, l'ignorance, l'indifférence et les intérêts [du capitalisme] cumulent leurs effets, ont des résultats plus graves encore qu'en métropole. ${ }^{73}$ » Daniel Lefeuvre, dans un ouvrage qui a soulevé une polémique, ou bien C. Bayly dans une étude récente sur la modernité ne soulignaient pas autre chose $^{74}$ : ce serait bien méconnaître le XIX ${ }^{\mathrm{e}}$ siècle, ce serait sous-estimer les problèmes sociaux de la métropole française - pour ne pas parler du cas du Japon qui connaît deux guerres civiles de suite dans les années 1868-1877 - que de croire que les " puissances » auraient connu des régimes "pacifiés et policés", et auraient, en quelque sorte, 
inventé des procédés de violence ou des systèmes de gouvernance inédits, destinés par essence ou uniquement aux colonies et aux colonisés.

Enfin, il faut insister sur un troisième point en revenant sur ce qui est sans doute une caractéristique fondamentale des empires coloniaux modernes, à savoir qu'ils ont été construits en même temps que les États-nations en Europe ou au Japon. C'est même précisément la compétition entre les États-nations, plutôt que l'idée de nation, qui explique l'expansion coloniale : même les États-Unis ont participé à ce mouvement, bien qu'ils n'aient pas eu d'empire colonial au sens strict du terme pour des motifs idéologiques - l'idée que les États-Unis seraient un pays "libérateur", car une excolonie décolonisée via une guerre coloniale, est centrale dans la doctrine interventionniste américaine depuis la guerre (en réalité colonialiste) contre l'Espagne de 1898 pour le contrôle de Cuba et des Philippines, équivalent de l'argument " civilisateur » des autres puissances coloniales. Le cas de la Grande-Bretagne pourrait sembler rendre malaisée la distinction entre colonisation d'Ancien Régime (ou plutôt prémoderne; notamment le cas des dominions, qui étaient semi-autonomes) et colonisation moderne, mais il faut conserver à l'esprit deux éléments essentiels : 1) le poids de sa colonisation en Afrique et en Asie orientale au XIXe siècle ; 2) le fait que l'Empire des Indes ne fut consolidé au travers d'un système colonial systématique (i.e. directement contrôlé par l'État britannique) que consécutivement à la révolte des Cipayes de 1857-1858, c'est-à-dire dans la seconde moitié du XIXe siècle, participant ainsi du mouvement de colonisation moderne. En outre, bien qu'ayant des traits nationaux plus précoces, comme la France ou le Japon, il est impossible de parler d' 'État nation » dans le cas de la Grande-Bretagne avant les avancées de la Révolution industrielle et de la scolarisation au XIX ${ }^{e}$ siècle.

En effet, en ce long xIX siècle allant de la chute de l'Empire napoléonien jusqu'à la Première Guerre mondiale, qui vit les États centralisés se constituer des "armées nationales" et mettre en place, sous diverses formes toujours équivalentes ou homologues, des « cultes des soldats tombés à la guerre $»^{75}$, cette allégeance, ce sens du sacrifice pour l'État et même l'obligation en cas de conflit interétatique de se sacrifier pour l'État, tous ces éléments ont provoqué - en France et au Japon cela est très net un phénomène particulier : à savoir l'encouragement fait aux troupes coloniales (les indigènes) à « mourir pour la patrie ", c'est-à-dire à mourir pour la métropole. C'est pour cette raison que tant de soldats issus des colonies sont morts pour le Japon ou pour la France (il reste aujourd'hui environ 28000 Algériens encore vivants ayant participé à la Seconde Guerre mondiale), et c'est pour cela qu'il existe un débat autour de la célébration des troupes coloniales dans les lieux de cultes militaires nationaux susmentionnés ou bien autour des pensions aux anciens combattants coloniaux.

À bien y penser, cela est tout à fait compréhensible pour des pays qui étaient des puissances coloniales : à l'époque des empires coloniaux l'allégeance à l'État dépassait l'idée étroite de nationalité. Et c'est aujourd'hui, maintenant que les identités ont été «re-nationalisées », qu'un débat voit le jour sur l'engagement des colonisés au sein des conflits que mena leur ex-métropole coloniale. Il est vrai que le culte des soldats morts pour la patrie en France n'est pas entaché par la présence de "criminels de guerre » comme c'est le cas au Japon, mais cela dérive uniquement du fait que le général De Gaulle a fait interdire tout procès concernant les «événements d'Algérie » via quatre lois passées en 1962, 1964, 1966 et 1968, elles-mêmes complétées par l'effacement des condamnations prononcées contre les membres de l'OAS en 1974, puis en 1982 par la 
réhabilitation de ses membres sur volonté de François Mitterrand ${ }^{76}$. C'est pour cette raison qu'il n'existe pas juridiquement de « criminels de guerre » en France.

\section{Pour conclure}

Comme nous avons tenté de le montrer, il est impossible d'interroger une "spécificité " japonaise, et plus généralement une spécificité nationale, tant pour la colonisation que les "guerres de mémoires" actuelles, qui sont dans les deux cas des phénomènes mondiaux. Cela d'une part parce que les colonisations modernes doivent être rapprochées autour d'une dynamique commune et unique dérivant de la compétition interétatique entre les puissances, et, d'autre part, parce que le décalage mémoriel Europe/Japon s'explique plutôt par une décolonisation achevée plus tôt au Japon. Ce décalage est, aujourd'hui, renforcé par une situation géopolitique totalement différente et indépassable : l'Algérie n'est pas la $11^{\mathrm{e}}$ puissance économique mondiale, comme la Corée du Sud, et derrière elle l'Afrique ne constitue pas un État unifié de 1,3 milliard d'habitants doté de l'arme nucléaire, comme l'est la République populaire de Chine ; en outre, la France a besoin du gaz algérien. Le Japon se voit placé de ce fait dans une situation plus ardue que celle de la France vis-à-vis de son passé et de ses crimes coloniaux, alors même que la France est le pays d'Europe occidentale qui a pourtant le plus violé les Droits de l'Homme après 1945. On pourrait même considérer que la France a été en guerre de façon continue de 1939 à 1962. Ce décalage se comble aujourd'hui, depuis 2000. Pour évoquer la Grande-Bretagne, la transition britannique «pacifique » est un mythe qui masque la répression en Inde durant la Seconde Guerre mondiale, féroce à partir de 1942, la grande famine du Bengale de 1943, et surtout les guerres menées en Afrique, notamment au Kenya, dans les années 1950.

Car, pour être rigoureux, la question de la mémoire devrait nous éloigner des a priori voire des polémiques sur une " culture coloniale », pour plutôt nous ramener à l'étude de l'histoire de la colonisation elle-même. Et pour que celle-ci soit pleinement analysée, le Japon ne peut raisonnablement pas être exclu, ou traité comme un cas « spécifique ", $\mathrm{du}$ grand mouvement colonialiste $\mathrm{du} \mathrm{xIx}^{\mathrm{e}}$ siècle, dont il participa en synergie avec les autres puissances. La question de la place de la Chine dans les représentations collectives de l'époque, ainsi que les trois facteurs évoqués en dernière partie afin de rapprocher ces colonisations croisées - la durée, la distance et la superposition entre la période de mise en place de l'État-nation et la phase de construction de l'Empire colonial - nous semblent centraux pour réfléchir sur le xix et la première moitié du xxe siècle.

En mettant ces quatre facteurs au centre de notre réflexion, peut-être sera-t-il possible de dépasser les limites d'un débat historique et politique - aujourd'hui en réalité mondialisé - qui a toujours choisi d'insister sur la « spécificité » japonaise (ou française, via un "occidentalisme » qui ne serait qu'un orientalisme inversé), tant chez les historiens européens qu'est-asiatiques, et même japonais comme on l'a noté, pour revenir sur l'histoire du partage du globe entre les puissances à la fin du XIX ${ }^{e}$ siècle. Autant de dynamiques globales qui interdisent toute réduction du phénomène colonial à l'Occident, ou de la violence au Japon, mais soulignent plutôt des logiques et ambitions étatiques ainsi qu'une volonté de puissance chez ceux qui participèrent du «nationalisme des forts» (en opposition aux nationalismes nés en réaction à la colonisation), tout au long de l'ère des empires. 


\section{NOTES}

1. Éric Hobsbawm, L’Ère du capital, 1978 [1975], Paris, Hachette, 2002, p. 240.

2. Pour la France, de façon exemplaire avec une guerre de « recolonisation " en Indochine dès 1945, puis en 1954 une opération de « pacification » dans ses départements d'Algérie. On revient sur le cas de la Grande-Bretagne plus bas.

3. Notamment, la question des «femmes de réconfort » de l'armée impériale, surtout coréennes, ou celle des travailleurs forcés coréens déportés au Japon à la fin des années 1930 - population distincte des migrants des années 1920 -, concernent la Seconde Guerre mondiale et non la colonisation. De même, le retour de ces déportés en Corée après 1945 relève du traitement de l'après-guerre. Voir A. Nanta, "Japon ", in Pierre-André Taguieff (dir.), Dictionnaire critique du racisme, Paris, Fayard, 2009.

4. Celle-ci n'a bien sûr jamais existé, la guérilla perdurant partout de façon endémique.

5. Concernant ces débats touchant à la mémoire et aux responsabilités étatiques, il faut souligner l'impossibilité de superposer " polémiques historiques » et « lieux de justice », car les dispositifs juridiques n'ont pas la capacité de clore les controverses historiques ou mémorielles comme l'ont montré les «lois mémorielles» en France: loi sur le génocide arménien et loi pour la reconnaissance de l'esclavage de 2001, ou loi du 23 février 2005 qui affirma que la colonisation française était " positive » et voulait contraindre enseignants et chercheurs à suivre cette voie. (De façon semblable, durant la colonisation, la légalité mise en avant par l'État colonisateur en Algérie, au Viêtnam ou en Corée, ne lui conférait pas de légitimité autre que la sienne).

6. Sur la colonisation japonaise, qui continue, elle aussi une colonisation d'Ancien Régime (à Hokkaidō et, sous une autre forme, à Okinawa), il existe de nombreuses publications universitaires remontant aux années 1960 (cf. infra partie 1). Voir notamment : Pierre-François Souyri, «La colonisation japonaise : un colonialisme moderne, mais non occidental », in Marc Ferro (dir.), Le Livre noir du colonialisme, (1 ${ }^{\text {re }}$ éd. 2003), Paris, Hachette, 2005, p. 543-574 ; Gi-Wook Shin et Michael Robinson (dir.), Colonial Modernity in Korea, Harvard, Harvard University Press, 1999; Oguma Eiji 小熊英二, “Nihonjin” no kyōkai「日本人」の境界 (Les frontières des «Japonais »), Tōkyō, Shin.yō-sha 新曜社, 1998 ; Michel Vié, Le Japon et le monde au Xxe siècle, Paris, Masson, 1995 ; Pierre-François Souyri, «Une forme originale de domination coloniale? Les Japonais et le Hokkaidō avant l'époque Meiji », in Martine Godet (dir.), De Russie et d'ailleurs, Mélanges Marc Ferro, Paris, Institut d'études slaves, 1995, p. 373-388; Ōe Shinobu 大江志乃夫 (dir.), Kindai Nihon to Shokuminchi 近代日本と植民地 (Le Japon moderne et ses colonies), Tōkyō, Iwanami shoten 岩波書店, 1992-1993, 8 vols.; Ramon H. Myers et Mark R. Peattie (dir.), The Japanese Colonial Empire, 1894-1945, Princeton, Princeton Univ. Press, 1984 ; Kobayashi Hideo 小林 英夫, “Daitōa kyōei ken” no keisei to hōkai 「大東巠共栄圈」の形成と崩壊 (Genèse et chute de la “Sphère de co-prospérité de la Grande Asie Orientale), Tōkyō, Ochanomizu shobō 御茶ノ水書房, 1975, édition révisée 2006.

7. Sur l'émergence de l'historiographie française de la colonisation française, et les débats mémoriels associés, voir l'article de synthèse: Claude Liauzu, «Entre mémoires et histoire: controverses sur les enjeux du passé colonial », Matériaux pour l'histoire de notre temps, $\mathrm{n}^{\circ} 85$, Paris, BDIC, 2007, p. 27-32. Voir aussi les remarques de Bernard Droz, en préface à : Jacques Dalloz, Dictionnaire de la Guerre d'Indochine, Paris, Armand Colin, 2006, p. 5-8. Si l'historiographie de l'Algérie coloniale connait aujourd'hui une augmentation, les travaux nouveaux se concentrent cependant sur la Guerre d'Algérie.

8. Sur ces débats et sur la mémoire de la guerre et de la colonisation au Japon: A. Nanta, «Le Japon face à son passé colonial », in Olivier Dard et Daniel Lefeuvre (dir.), L'Europe face à son passé colonial, Paris, Riveneuve éditions, 2008, p. 129-146. 
9. Inoue Kiyoshi, Nihon no gunkokushugi, Tōkyō, Tōkyō daigaku shuppankai, 2 vols., 1953 ; Tennōsei, Tōkyō, Tōkyō daigaku shuppankai, 1953. La superposition entre Tennō-sei et impérialisme apparaît au mieux dans sa synthèse de 1968 : Inoue, Nihon teikokushugi no keisei 日本帝国主義の 形成 (La formation de l'impérialisme japonais), Tōkyō, Iwanami shoten, 1968.

10. Pas forcément nostalgique : sur la colonisation en Corée, on pourra citer le roman critique d'Ikeyama Hiroshi 池山廣, Nihon no kiba 日本の牙 (Les crocs du Japon), Tōkyō, Tōga shobō 冬芽 書房, 1950 .

11. Kobayashi a débuté son histoire économique d'abord en rapport avec l'Empire colonial avant d'étudier ensuite ses caractéristiques lors de la Seconde Guerre mondiale (1975, op. cit.). Cette seconde recherche ne peut être comprise chez lui qu'en rapport avec la première. Pour un ouvrage ancien : Yamada Hideo 山田秀雄 (dir.), Nihon shokuminchi keizai shi no sho mondai 日本植 民地経済史の諸問題 (Problèmes concernant l'histoire économique des colonies [japonaises]), Tōkyō, Ajia keizai shuppan kai アジア経済出版会, 1973.

12. Yamabe Kentarō, Nikkan heigō shōshi 日韓併合小史 (Petite histoire de la Fusion nippocoréenne), Tōkyō, Iwanami shoten, 1965.

13. Yamabe Kentarō, Nihon tōchi-ka no Chōsen, Tōkyō, Iwanami shoten, 1971.

14. La question des pressions occidentales au $\mathrm{XIX}^{\mathrm{e}}$ siècle était jusque-là le grand sujet des historiens marxistes, et ce tournant ne doit donc pas être sous-estimé.

15. Ōe Shinobu, Kindai Nihon to Ajia, Tōkyō, Sanseidō, 1968.

16. Il s'agit de la collection de référence : Ōe Shinobu (dir.), Kindai Nihon to shokuminchi, op. cit.

17. Ichikawa Masaaki 市川正明 (dir.), Kankoku heigō shiryō 韓国併合資料 (Archives relatives à l'annexion de la Corée), Tōkyō, Hara shoten 原書店, 3 vol. , 1978.

18. Iwanami kōza Nihon rekishi 岩波講座 日本歴史, Tōkyō Iwanami shoten, 1962-1964 et 1975-1977, vingt-cinq volumes pour chaque édition. La première édition (en 130 fascicules) date de 1933-1935.

19. Claude Liauzu, « Entre mémoires et histoire... », op. cit.

20. Pai Hyung-Il (Pae Hyǒng-Il) a souligné encore récemment l'importance de Hatada dans la critique, dès les années 1960, de l'historiographie coloniale (i.e. de la période coloniale). Pai Hyung-Il, Constructing "Korean" Origins. A Critical Review of Archaeology, Historiography, and Racial Myth in Korean State-Formation Theories, Harvard, Harvard University Press \& Hallym, 2000, p. 425, note 22.

21. Sur le régime d'après 1945, voir : Michael Lucken, Anne Bayard-Sakai et Emmanuel Lozerand (dir.), Le Japon après la guerre, Paris, Philippe Picquier, 2007.

22. L'historien libéral Maruyama Masao 丸山眞男 (1914-1996) a largement contribué à répandre ce schéma du côté japonais. On le retrouve par exemple cité par Benedict Anderson, dans L'Imaginaire national (édition augmentée, Paris, La Découverte, 1996, p. 105, et note 30 en p. 105) quand Anderson tente d'expliquer la différence entre l'idée de nation en Europe et au Japon, et la nature «agressive» de celui-ci. Cependant, Maruyama ne suivait pas les historiens marxistes dans leur critique de la modernité et de l'État de Meiji, et considérait nécessaire de revenir au projet modernisateur du XIX ${ }^{\mathrm{e}}$ siècle, qu'il considérait avoir dérapé dès 1894. Jacques Joly, «Maruyama Masao : de l'autonomie au pacifisme », in M. Lucken et al. (dir.), op. cit., p. 85-108. Sur l'historiographie japonaise et les débats entre historiens : A. Nanta, «Débats et tensions autour du passé dans le Japon d'après 1945 », in Christian Delacroix, Patrick Garcia, François Dosse et Nicolas Offenstadt (dir.), Historiographies, concepts et débats, Paris, Gallimard, collection Tel, 2009 ; Tōyama Shigeki 遠山茂樹, Sengo no rekishigaku to rekishi ishiki 戦後の歴史学と歴史認識 (Historiographie et conscience historique après-guerre), Tōkyō, Iwanami shoten, 1969 ; Oguma Eiji, “Minshu” to “aikoku”「民主」と「愛国」 (Démocratie et patriotisme), Tōkyō, Shin.yō-sha, 2002; Nagahara Keiji 永原慶二, 20 seiki Nihon no rekishigaku 20世紀日本の歴史学 (L'historiographie japonaise au Xx siècle), Tōkyō, Yoshikawa kōbunkan 吉川弘文館, 2003. 
23. Cette lecture fait l'impasse sur la longue histoire de la démocratie au Japon, remontant aux années 1880 et ayant débouché sur le suffrage universel masculin en 1925 (ou bien ne se concentre que sur les années 1920), autrement dit sur une culture du parlementarisme au Japon qui n'a pas du tout été « apportée " par les États-Unis après 1945.

24. Edward W. Saïd, L'Orientalisme : l'Orient créé par l'Occident, 1978, Paris, Seuil, 1980, 2005.

25. De même, le déroulement concret des opérations militaires (les crimes de guerre et les crimes contre l'Humanité, soit les catégories B et C) ne fut pas discuté lors du Procès de Tōkyō (Tribunal militaire international pour l'Extrême-Orient), hormis le cas du massacre de Nankin en décembre 1937, avec donc une ambiguïté dans le cas du général Matsui Iwane 松井石根 (1878-1948). Franck Michelin, «Le procès des criminels de guerre japonais ", L'Histoire, $n^{\circ} 271,2002$, p. 54-61.

26. Voir ci-dessous, ainsi que l'article de Takahashi Tetsuya «Les militaires coréens de l'armée japonaise et les procès pour crimes de guerre » dans le présent numéro.

27. Sur ce dernier point, cela a changé depuis 2000 et l'apparition d'un discours sur la « république coloniale». L'amnésie française vis-à-vis de la colonisation et de la guerre en Indochine est remarquable, du fait que cette partie de l'empire était plus éloignée de la métropole que l'Algérie ou la Corée.

28. Kim Ku 金九 (1876-1949) puis, après 1948, le Président Yi Sŭngman 李承晚 (1875-1965) souhaitaient participer au Procès de Tōkyō et aux négociations du Traité de San Franscisco (1951), et faire reconnaître la Corée comme "pays belligérant", en s'appuyant sur une «déclaration de guerre » émise par le Gouvernement provisoire (à Shanghai) à l'encontre du Japon en décembre 1941. Les États-Unis et la Grande-Bretagne s'y opposèrent afin de ne pas créer de précédent fâcheux dans un ordre mondial encore largement colonial.

29. Sur l'oubli post-décolonisation, voir: Benjamin Stora, La Gangrène et l'oubli, Paris, La Découverte, 1991, réédité en 2005. Cet ouvrage est en outre très critique à l'encontre de l'historiographie algérienne.

30. Les excuses et réparations italiennes à l'encontre de la Lybie, en 2008, constituent bien un précédent. Il faut cependant prendre en compte la nature des accords bipartites passés à cette occasion, et le fait que l'Italie n'a traité qu'avec la Lybie.

31. En raison du rôle de l'armée du Guómíndăng, et du fait de la présence de Jiang Jièshi 蒋介石 (Tchang Kaï-Chek ; 1887-1975) lors de la Déclaration du Caire, le $1^{\text {er }}$ décembre 1943. Ces données sont essentielles pour définir la position réelle de la Chine, mais à la fin de la Seconde Guerre mondiale.

32. M. Vié, Le Japon et le monde au $\mathrm{XX}^{e}$ siècle, op. cit., chap. 7, notamment p. 164-165.

33. Christopher A. Bayly, La Naissance du monde moderne (1780-1914), 2004, Paris, Éditions de l'Atelier, 2007, p. 374-375.

34. En outre, les quatorze points de Wilson (8 janvier 1918) avaient été pensés afin de régler « la question nationale » en Europe centrale, c'est-à-dire le problème des populations enjeux de luttes entre les puissances dans cette région, et non pour donner la parole aux colonisés, bien qu'ils y fussent mentionnés et quelles que fussent les intentions de Wilson. Marc Ferro, La Grande Guerre, Paris, Folio, 1969, p. 378-385.

35. M. Vié, Le Japon et le monde au $\mathrm{Xx}^{e}$ siècle, op. cit.; R. Myers et M. Peattie (dir.), The Japanese Colonial Empire, 1894-1945, op. cit., introduction. Ces ouvrages diffèrent cependant en ce que le second ne relie pas l'expansion du début des années 1930 avec l'empire colonial «formel », à la différence de l'analyse de M. Vié, qui considère «l'incident de Mandchourie " comme l'achèvement du processus de colonisation (cf. supra). Cependant, Mark Peattie est loin d'oublier la question du rapport de la Mandchourie à l'Empire japonais, même s'il distingue Empire «formel» (colonial ?) et «informel» (non colonial ?), distinction pas forcément pertinente, comme nous l'expliquons ici (l'Algérie n'était administrativement pas plus une colonie que ne l'était la Corée). P. Duus, R. Myers et M. Peattie (dir.), The Japanese Informal Empire in China, 1895-1937, Princeton, Princeton Univ. Press, 1989. 
36. Kojong portait ce titre depuis 1897.

37. Rappelons que le Traité de commerce imposé par le Japon à la Corée en 1876 se situe entre l'occupation de l'île Kanghwa par la France en 1866 (qui donna lieu à un pillage des archives royales coréennes, jamais rétrocédées), l'incident Kanghwa avec les États-Unis en 1871 et l'occupation de l'île Kǒmun par l'Angleterre en 1885-1887.

38. Ce double discours dans les représentations est déjà présent à cette époque, comme l'a montré $\mathrm{M}$. Vié en soulignant le caractère comparativement tardif de la critique vis-à-vis de l'Allemagne nazie (Le Japon et le monde au Xxe siècle, op. cit., p. 145).

39. En outre, ce type de discours invoque l'idée d'une proximité « raciale » ou culturelle entre dominants et dominés en Asie, ce qui était justement le discours de l'idéologie annexionniste de l'époque.

40. À ce sujet, voir: Immanuel Wallerstein, L'Universalisme européen. De la colonisation au droit d'ingérence, 2006, Paris, Demopolis, 2008, p. 46 et suiv., p. 54-55 et suiv.

41. Discours qui, dans un premier temps, avait été approuvé par l'opinion publique française à plus de $60 \%$, à propos de la loi du 23 février 2005. Cf. infra sur l'impossibilité des excuses.

42. On renvoie à notre conférence du 9 février 2007 à la Maison franco-japonaise : «Les débats sur la légitimité de l'annexion de la Corée ». Nous publierons prochainement un article sur cette question.

43. En japonais : inochi o sasageta 命を捧げた.

44. Voir: George L. Mosse, Fallen Soldier, Oxford, Oxford university Press, 1990 ; Takahashi Tetsuya 高橋哲哉, Yasukuni mondai 靖国問題, Tōkyō, Chikuma shobō 筑摩書房, 2005, trad. française Arnaud Nanta, Les Belles Lettres, 2012.

45. Il ne s'agit pas, pour nous, de reprendre la thèse de Pascal Blanchard (par ex. dans La Fracture coloniale, Paris, La Découverte, 2005 ; cet ouvrage contient des contributions variées qui ne peuvent être réduites à la position de P. Blanchard) ou des «Indigènes de la République », qui affirment que l'idée de nation serait par essence colonialiste et que, par conséquent, la France (ou par extension les autres anciennes puissances) seraient toujours, après la décolonisation, des États par essence coloniaux, les actuels problèmes de mémoire étant ainsi expliqués. Nous essayons, pour notre part, de comprendre les conflits mémoriels en posant une certaine continuité dans les représentations de l'autre, ce qui n'est pas l'équivalent de la position déjà mentionnée, qui méprise l'historicité de la colonisation moderne et n'accorde pas beaucoup d'importance au fait que des pays soient colonisés ou devenus indépendants.

46. Aimé Césaire assimilait le nazisme et la colonisation dans son Discours sur le colonialisme : «Ce qu'il [le bourgeois européen] ne pardonne pas à Hitler, ce n'est pas le crime en soi, le crime contre l'homme, c'est le crime contre l'homme blanc, c'est l'humiliation de l'homme blanc, et d'avoir appliqué à l'Europe des procédés colonialistes dont ne relevaient jusqu'ici que les Arabes d'Algérie, les coolies de l'Inde et les nègres d'Afrique ». (1950, Présence africaine, 1994, p. 12).

47. Voir par exemple : B. Stora, La Guerre des mémoires, Paris, Ed. de l'Aube, 2007. Bien que l'auteur affiche une volonté certaine de prise de distance, le propos de l'ouvrage est proche d'un ouvrage récent de Daniel Lefeuvre (D. Lefeuvre, Pour en finir avec la Repentance coloniale, Paris, Flammarion, 2006) en ce qu'il oppose mémoires revendicatrices et pratique historienne.

48. La Gauche japonaise n'ayant plus un poids politique important, sa place dans ce débat doit néanmoins être relativisée.

49. Sur les réactions de Pierre Moscovici, de Jean-Marc Ayrault ou de Julien Dray: "Le climat s'alourdit entre Paris et Alger avant la visite d'État de Nicolas Sarkozy », Le Monde, 29 novembre 2007 ; «M. Moscovici (PS) ne veut pas que la France "verse dans l'autoflagellation" », Le Monde, 3 décembre 2007. S'il s'agissait du Japon, l'extrême droite serait immédiatement évoquée.

50. À propos du précédent italien, voir la note 30 .

51. A. Nanta, «Le Japon face à son passé colonial », op. cit.

52. Bernard Droz, Histoire des décolonisations au $\mathrm{XX}^{e}$ siècle, Seuil, 2006. 
53. Ibid., p. 8 et p. 20-21. Il ne s'agit pas ici de critiquer cet ouvrage, dont la qualité n'est pas en question, mais plutôt de souligner le traitement différentiel dont fait l'objet la colonisation japonaise au sein de l'historiographie occidentale, ici dans un ouvrage récent.

54. Oguma, "Nihonjin" no kyōkai, op. cit.

55. Voir la discussion récente par Yves Lacoste dans la revue Hérodote, $\mathrm{n}^{\circ} 128,2008$, rubrique «Hérodote a lu», p. 157-168. C. Bayly estime même que l'Angleterre a pratiqué des formes de gouvernance tout à fait centralisées dans ses colonies, formes qui eussent été impossibles en métropole (op. cit.).

56. Même dans les départements d'Algérie la loi était dérogatoire, en réalité régie par des décrets du gouverneur général ou du président de la République, et complétée par le statut de l'Indigénat (qui ne fut jamais vraiment aboli avant 1962). Le département d'Okinawa (1879) constitue un cas similaire.

57. Sur les velléités de l'empereur vis-à-vis de l'Algérie dans les années 1860, voir : Daniel Rivet, Le Maghreb à l'épreuve de la colonisation, Paris, Hachette, 2002, p. 130-133. Il faut aussi évoquer la proposition par l'homme politique Maurice Viollette (1870-1960) dans les années 1930 d'élargir le droit de vote en Algérie aux "évolués » (c'est-à-dire aux indigènes culturellement assimilés, selon le mot d'alors), proposition timide qui n'a cependant pas été écoutée. Il semble dès lors difficile d'associer colonisation française et assimilation politique. En outre, notons que Viollette prit la défense de l'empire français après 1945, durant les guerres coloniales.

58. Voir par exemple : Marc Ferro (dir.), Le Livre noir du colonialisme, op. cit. On peut se demander ce que désigne le néologisme "hyper-nationalisme » de B. Droz à propos du Japon ; Histoire des décolonisations au $\mathrm{XX}^{e}$ siècle, op. cit.

59. Voir: Daniel Hémery et Pierre Brocheux, Indochine, la colonisation ambiguë, Paris, La Découverte, édition augmentée, 2001, p. 306-309 ; Sakano Tōru 坂野徹, « Hōki no konseki. Musha jiken to Taiwan ni okeru minzoku shinrigaku kenkyū o megutte» 蜂起の痕跡霧社事件と台湾に おける民族心理学研究をめぐって (Traces d'une insurrection : les études sur la psychologie des ethnies taiwanaises et sur l'incident de Wùshè), in Kurokawa Midori 黒川みどり, « Manazasareru mono » no kindai 眼差させる者の近代 (La modernité des « personnes observées »), Tōkyō, Kaihō shuppansha 解放出版社, 2007, p. 219-250.

60. P. F. Souyri, « La colonisation japonaise... », op. cit., p. 573.

61. Nous ne traitons pas ici de la colonisation d'Ancien Régime qui fonctionnait selon des logiques différentes, même si l'on ne peut sans doute pas l'opposer trop schématiquement à la moderne. La colonisation d'Ancien Régime servait les logiques étatistes des puissances des XVI $\mathrm{XVIII}^{\mathrm{e}}$ siècles, mais était largement déléguée à des compagnies à charte, sans que l'État ne s'impliquât directement et sans lien avec une nation n'existant alors pas (au moins comme entité politique consciente). Inversement, aux $\mathrm{XIX}^{\mathrm{e}}$ et $\mathrm{XX}^{\mathrm{e}}$ siècles, il eut été impensable que les nouvelles colonies de peuplement, telles l'Algérie ou la Corée, puissent être détachées de la métropole. Enfin, les territoires concernés n'étaient soit pas les mêmes (France, Japon, et même la GrandeBretagne pour l'Afrique et l'Asie), soit administrés différemment (la Grande-Bretagne en Inde après 1857-1858 en est une bonne illustration, cf. infra).

62. Ce point de vue a été largement défendu par Ramon Myers et Mark Peattie, qui ont été jusqu'à noter : « the Japanese colonial empire stands as an anomaly of modern history. Because it was assembled at the apogee of the "new imperialism" [i.e. la colonisation moderne] " Japanese Colonial Empire, 1894-1945, op. cit., p. 10). Comme nous le montrons dans la présente partie, cette affirmation se base sur une lecture biaisée de la colonisation moderne, dont l'apogée se situe autour de 1930 et non à la fin du XIX siècle. Concernant la Corée, voir le numéro thématique «Mémoires Europe-Asie » de la revue Vingtième siècle (2007-2, n 94) : cet argument y était une nouvelle fois présenté par des historiens coréens souhaitant rapprocher la Corée (que ceux-ci jugent ne jamais avoir été colonisée) de la France de Vichy (occupée). 
63. Sur l'expansion coloniale française en particulier, par exemple: Claude Liauzu (dir.), Colonisation: droit d'inventaire, Paris, Armand Colin, 2004; Marc Ferro (dir.), Le Livre noir du colonialisme, op. cit.

64. Comme l'a écrit un des premiers historiens français de la colonisation française, CharlesRobert Ageron : «Nommer les parlementaires actifs du parti colonial, c'est feuilleter le Gotha de la III ${ }^{\mathrm{e}}$ République ». C.-R. Ageron, France coloniale ou parti colonial ?, Paris, PUF, 1978, p. 75.

65. C. Liauzu (dir.), Colonisation: droit d'inventaire, op. cit.; M. Ferro (dir.), Le livre noir du colonialisme, op. cit. ; D. Rivet, Le Maghreb à l'épreuve de la colonisation, op. cit., chapitre 5 : « Le temps de l'Algérie française, 1870-1930», notamment p. 173-185. Les mouvements de résistance continuèrent donc bien après la réédition d'Abd El-Kader en 1847, déjà tardive si l'on considère qu'elle eut lieu dix-sept ans après la prise d'Alger.

66. Voir : D. Hémery et P. Brocheux, op. cit.

67. Hugues Tertrais, Atlas des guerres d'Indochine, 1940-1990, Paris, Éditions Autrement, Mémorial de Caen, Ministère de la Défense, 2004, p. 10.

68. B. Droz, Histoire des décolonisations au XXe siècle, op. cit., p. 141-142.

69. Éric Hobsbawm, L’Ère des empires, 1989 [1987], Paris, Hachette, 2002, p. 109.

70. C. Liauzu, «Gouvernement colonial », in C. Liauzu (dir.), Dictionnaire de la colonisation française, Paris, Larousse, 2007, p. 315-319, notamment p. 319. Encore en 1973, alors en poste à Madagascar pour diriger les forces françaises du sud de l'Océan indien, le général Bigeard écrivait : «A 10000 kilomètres de l'Hexagone, il faut d'abord compter sur nos propres effectifs parfaitement affûtés, possédant un esprit, capables de réagir vite et bien... ». Marcel Bigeard, Pour une parcelle de gloire, Paris, Plon, 1975, p. 445.

71. D. Rivet, Le Maghreb à l'épreuve de la colonisation, op. cit., p. 121. Sur les différents noms du régime de décrets dans le cas du Japon : Oguma, "Nihonjin" no kyōkai, op. cit., p. 16.

72. Hannah Arendt, Les Origines du totalitarisme, vol. 2 : L'Impérialisme.

73. Madeleine Rebérioux, La République radicale?, Paris, Seuil, 1975, p. 130.

74. D. Lefeuvre, op. cit., notamment les chap. 1 et 2 ; C. Bayly, op. cit., p. 434 et suiv.

75. G. Mosse, op. cit.; Takahashi Tetsuya, Yasukuni mondai, op. cit.

76. Sur ces lois interdisant tout procès relativement aux actes perpétrés en Algérie, voir: B. Stora, La Gangrène et l'oubli, op. cit.

\section{RÉSUMÉS}

Souvent considérée comme "spécifique", l'histoire de la colonisation japonaise doit être réévaluée et comprise comme partie d'une unique dynamique mondiale, à comparer à celle des autres empires coloniaux modernes.

Often considered "separate", the history of Japanese colonization should be reassessed and included as part of a single global dynamic, and compared to other modern colonial empires. 
INDEX

Index géographique : Chine, Corée

キーワード : shokumin 殖民, kokumin kokka 国民国家, sensō no kioku 戦争の記憶, kioku 記憶, teikoku shugi 帝国主義, bōryoku 暴力, Kankoku 韓国, Chūgoku 中国, Taiheiyō sensō 太平洋戦争 (1941-1945), Shōwa jidai 昭和時代 (1945-1989), shigakushi 史学史, rekishi 歴史

Index chronologique : Shōwa (1923-1945), guerre du Pacifique (1941-1945)

Mots-clés : colonisation, violence, guerre de mémoire, impérialisme, mémoire, travail de mémoire, État-nation

Keywords : China, Colonization, Historiography, History, Imperialism, Korea, Memory, Nationstate, Orientalism, War of the Pacific (1939-1945), Shōwa (1923-1945)

Thèmes : histoire, historiographie

\section{AUTEUR}

\section{ARNAUD NANTA}

Centre de recherches sur le Japon/CNRS 